\title{
Influence of liposomal drug entrapment on percutaneous absorption
}

\author{
M.G. Ganesan, N.D. Weiner, G.L. Flynn and N.F.H. Ho \\ College of Pharmacy. The University of Michigan, Ann Arbor, MI 48109 (U.S.A.) \\ (Received November 9th, 1983) \\ (Modified version received January 24th, 1984) \\ (Accepted February 24th, 1984)
}

\section{Summary}

Results of permeation experiments involving finite dose diffusion c:lls with hairless mouse skin as the membrane indicate that neither intact liposome: nor the phospholipid of which they are comprised diffuses across the skin. Lipoph lic drugs like progesterone and hydrocortisone, which are intercalated within th: bilayer structure of the phospholipid in multilamellar liposomes, seem to pass thr ough the skin with comparable facility to free drug (comparable mass transfer coe ficients). On the other hand, highly polar glucose entrapped in the aqueous compar ments of the liposome is poorly available for transport. The results of in vitro rel ease rate studies and theoretical calculations indicate that the very slight flux of lif osomally incorporated glucose seen experimentally is attributable to a slow releas 2 rate of glucose out of the liposome followed by relatively rapid skin permeation of the free solute. On the other hand, for hydrophobic progesterone and hydrocort isone the experimental results and supportive theoretical analysis suggest direct transfer of drug from liposome to the skin. Considering this mechanism and owing to increased soluble payloads of lipophilic drugs through liposomal incorporation, $\mathbf{n}$ ore total drug may be delivered through skin via liposomes relative to simple aqueous solutions.

\section{Introduction}

The potential application of liposomes (concentric bilayer lipid vesicle ;) as drug carriers has been recently reviewed (Patel and Ryman, 1981; Poste, 1933). Most

Correspondence: N.D. Weiner, College of Pharmacy, University of Michigan. Aun Arbor, MI 48109, U.S.A. 
studies have been directed towards the use of liposomes as drug carriers in oral or parenteral administration. Use of liposomes for topical applications has received little attention until recently. Mezei and Gulasekharam (1980, 1982). Mezei and Singh (1983), and Schaeffer and Krohn (1982) reported the potential use of liposomes in topical applications for the skin and eyes. These reports indicate that liposomes facilitate the passage of drugs through skin and it has even been suggested by Mezei and Gulasekharam (1980) that the liposomes traverse the skin and act as drug carriers. This suggestion has been received critically as it is difficult to envision how particles as large as liposomes can diffusionally pass through the skin's dense horny layer.

Therefore, we have undertaken some range finding studies to determine the extent to which liposomes promote the permeation of chemicals through skin, the possible mechanisms involved in permeation and, further, to assess whether liposomes themselves pass through the skin harrier. A quantitative and mechanistic approach is taken.

\section{Experimental}

\section{Materials}

Dimyristoylphosphatidylcholine (DMPC), dipalmitoylphosphatidylcholine (DPPC), distearoylphosphatidylcholine (DSPC), stearylamine (SA), dicetylphosphate (DCP) and cholesterol (CHOL) were purchased from Sigma Chemicals (St. Louis, MO). Cholesterol was purified by recrystallization 3 times from ethanol solution; all other lipids were used without further purification. Hydrocortisone was supplied by Upjohn (Kalamazoo, MI). Progesterone was purchased from Aldrich Chemicals (Milwaukee, WI). $\left[{ }^{14} \mathrm{C}\right] \mathrm{Glucose}(5 \mathrm{mCi} / \mathrm{mmol}),\left[{ }^{3} \mathrm{H}\right]$ hydrocortisone $(95.2$ $\mathrm{Ci} / \mathrm{mmol})$. [ $\left.{ }^{3} \mathrm{H}\right]$ progesterone $(55 \mathrm{Ci} / \mathrm{mmol})$ and $\left[{ }^{14} \mathrm{C}\right] \mathrm{DPPC}(115 \mathrm{mCi} / \mathrm{mmol})$ were purchased from New England Nuclear (Boston, MA). All other chemicals were of reagent grade. The lipids were dissolved in chloroform and stored under nitrogen at $-20^{\circ} \mathrm{C}$. Normal saline from Abbott Labs. (N. Chicago, IL) was used. Water was triply distilled in an all-glass still.

\section{Preparation of liposomes}

The lipid in chloroform solution was transferred to $10 \mathrm{ml}$ round-bottom flasks and the solvent was evaporated with nitrogen. Hydrocortisone or progesterone $(0.1 \%$ final concentration) with or without radio-tracer was also dissolved in chloroform and dried with the lipid in the same flask. The flask was rotated by hand to produce a thin layer of lipid at the bottom of the flask. The flask was left overnight under vacuum to remove the residual solvent. The lipid film was then suspended at the phase transition temperature of the saturated lecithin component in a sufficient amount of normal saline by vortexing for $10 \mathrm{~min}$ to yield a final concentration of about $65 \mu \mathrm{mol}$ lipid per $\mathrm{ml}$. For experiments involving glucose, normal saline containing radiolabeled glucose $(2 \mu \mathrm{Ci})$ was added. In some studies stearylamine or dicetylphosphate (10 mole\%) was added to give a net positive or negative charge to 
the liposomes. In yet another study liposomes were prepared with 30 molef cholesterol. The liposomal suspensions were centrifuged 3 times for $20 \mathrm{~min}$ at $\mathbf{1 0 . 0 0 0}$ rpm. Each time the supernatant was removed to discard small particles ind the liposomal pellet resuspended in fresh normal saline. The washed liposomal jreparation was then used for subsequent experiments. No determination of final solute to lipid ratio was performed. Liposomes thus formed were found to be multilar rellar as determined by negative stain electron microscopy.

The volume fraction of all liposome preparations, determined by centri uging 1 $\mathrm{ml}$ of the liposomal suspension in a graduated centrifuge tube at $10,000 \mathrm{rp}$ I for 60 min, was found to be approximately 0.075 . The average size of blank liposo nes was $0.8 \mu \mathrm{m}$ in diameter (range of $0.4-1.0 \mu \mathrm{m}$ ) as determined by negative stair. slectron microscopy.

\section{Differential scanning calorimetry}

Thermograms were obtained using a Perkin Elmer differential scanning $\mathrm{c}$ ulorimeter (Model DSC-2C) with thermal analysis data station for data analysis. Lif osomes were prepared as previously described. Samples were placed in sample $r$ ans for volatile matter and were properly sealed. Each sample was usually $16 \mu 1$ and contained approximately $1 \mu$ mole lipid. An equal amount of buffer was placed in the reference pan. All the scans were obtained at a heating rate of $5^{\circ} \mathrm{C} / \mathrm{min}$ and a range setting of $1 \mathrm{mcal} / \mathrm{s}$. Indium standard and water were used to calibrate the c: lorimeter.

\section{In vitro release rates of entrapped solute}

About $5 \mathrm{ml}$ of liposomal suspension containing entrapped solutes were frepared in a manner described earlier. Exactly $200 \mu \mathrm{l}$ samples were obtained with t me and centrifuged for $60 \mathrm{~min}$ at $10,000 \mathrm{rpm}$. The supernatant liquid was assaved b; liquid scintillation counting (Beckman LS 9000).

\section{Skin permeation studies}

Sixty-day-old, male hairless mice (SKH hr-1 strain) supplied by Skin and Cancer Hospital (Philadelphia, PA) were used. Appropriate size sections of full-llickness skin were excised from the abdominal surface and mounted in Franz diffusion cells (Crown Glass, Somerville, NJ). The diffusional cross-sectional area of the : sin was $0.785 \mathrm{~cm}^{2}$. A $200 \mu$ l liposomal suspension was placed on the skin in th. donor compartment and the receiver compartment was filled with $5.2 \mathrm{ml}$ normal sa ine and was well stirred. Water at $37^{\circ} \mathrm{C}$ was circulated throughout the water jackst of the diffusion cell. Microliter samples were withdrawn f.om the receiver comp irtment every hour for a $60-\mathrm{h}$ period and counted in the liquid scintillation spectror teter.

From the plots of the concentration of permeant appearing in the $r$ reiving chamber with time, the permeability coefficients were calculated. The flux of mass from the donor compartment is equal to the appearance rate of mass in the : eceiver.

$$
J=A P_{e} C_{D}=V \cdot \frac{d C}{d t}
$$


where $J$ is flux, mass $/ \mathrm{h}$; $A$ is area of skin, $\mathrm{cm}^{2} ; P_{\mathrm{e}}$ is effective permeability coefficient, $\mathrm{cm} / \mathrm{h} ; \mathrm{C}_{\mathrm{D}}$ and $\mathrm{C}$ are the drug concentrations in the donor and receiver chambers, respectively, mass $/ \mathrm{cm}^{3} ; \mathrm{V}$ is volume of receiving chamber, $\mathrm{cm}^{3}$ : and $\mathrm{t}$ is time, $h$. Experimentally, $C_{D}$ does not change appreciably during the experimental period; therefore, sink conditions in the receiver side essentially prevail. Consequently, the effective permeability coefficient is determined by

$P_{e}=\frac{V}{A C_{D}}\left(\frac{d C}{d t}\right)$

\section{Results}

Thermograms for the DPPC blank liposomes and liposomes containing glucose hydrocortisone and progesterone are shown in Fig. 1. The entrapment of glucose did not effect the baseline thermogram of DPPC liposomes. Hydrocortisone and progesterone, when incorporated in the liposomes, lowered the main transition temperature by $1-2^{\circ} \mathrm{C}$ and also eliminated the pretransition peak at $36^{\circ} \mathrm{C}$.

The in vitro release kinetics of glucose, hydrocortisone and progesterone from DPPC liposomes are shown in Fig. 2. It is seen that there was an initial rapid release

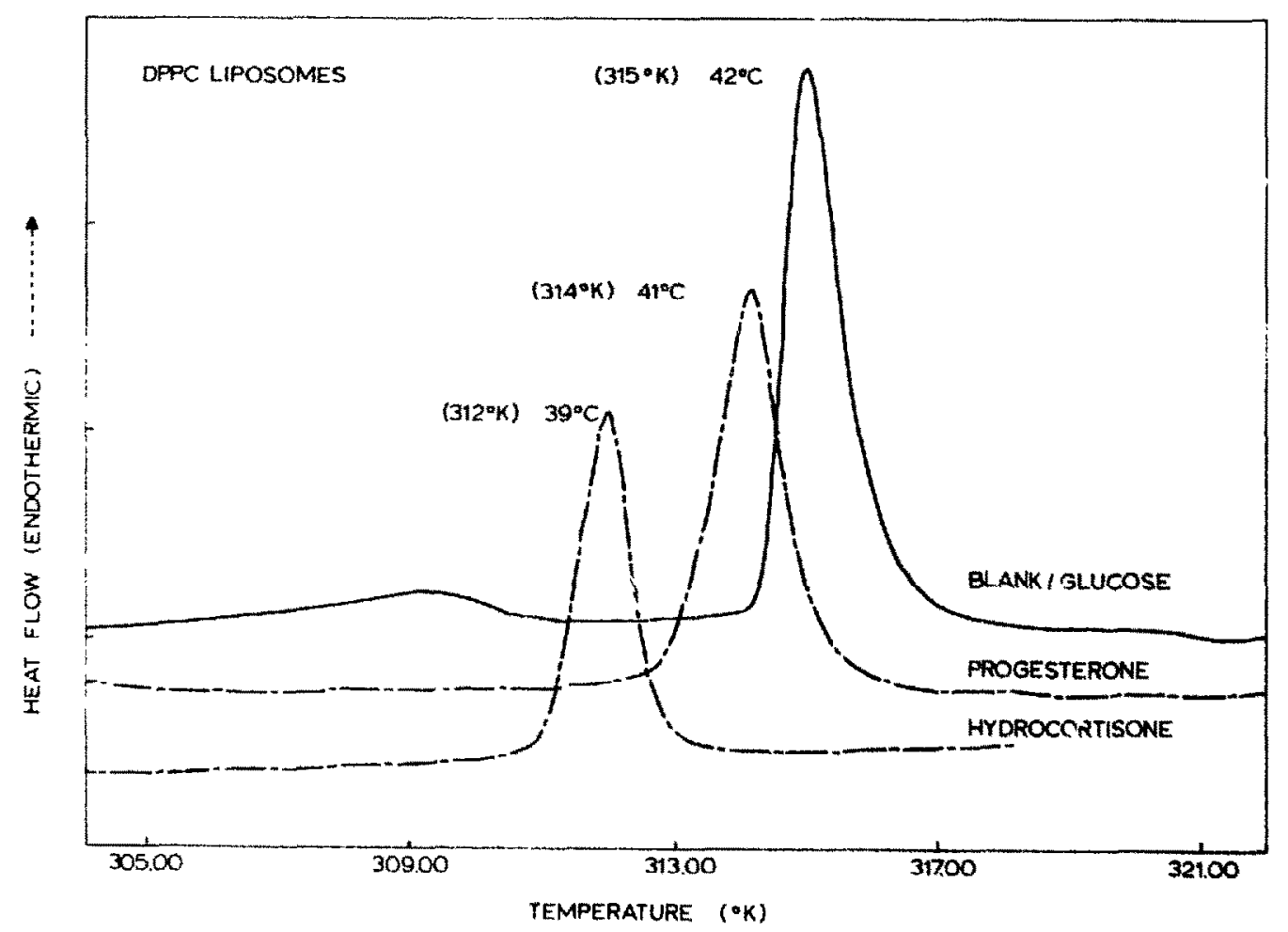

Fig. 1. Thermograms of multilamellar DPPC liposomes in the absence or presence of entrapped glucose. hydrocortisone and progesterone. 


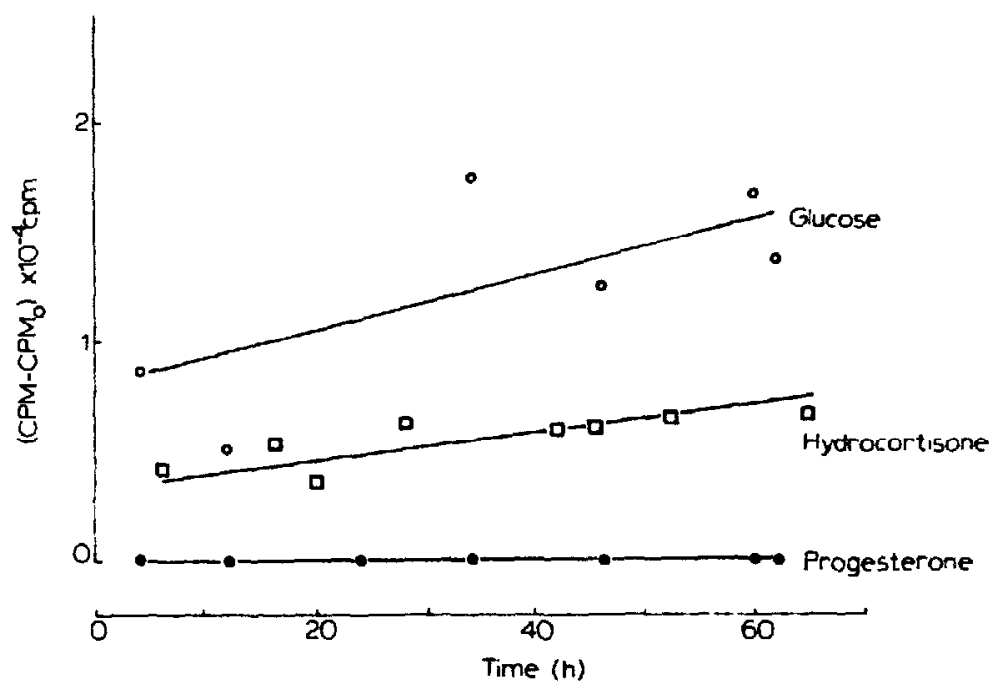

Fig. 2. In vitro release kinetic plots of glucose. hydrocortisone and progesterone from DPPC lposomes.

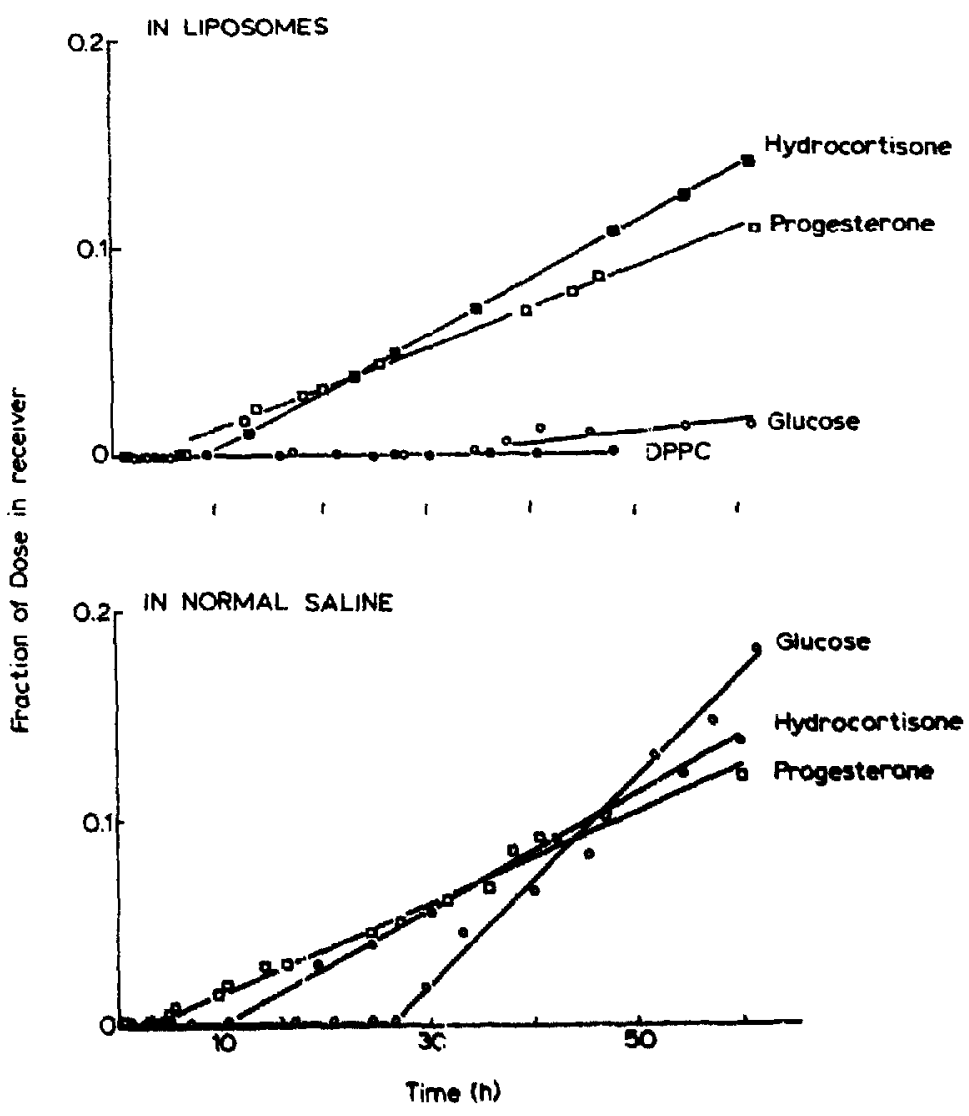

Fig. 3. Fraction of permeants appearing in the receiver compartment with time from solutes in : ormal saline solutions and liposomal suspensions. 


\section{TABLE 1}

PERMEABILITY COEFFICIENTS OF MODEL SOLUTES IN NORMAL SALINE AND DPPC LIPOSOMES

\begin{tabular}{|c|c|c|c|}
\hline \multirow[t]{2}{*}{ System } & \multicolumn{3}{|c|}{ Permeability coefficients $\times 10^{4}(\mathrm{~cm} / \mathrm{h})($ S.D. $)$} \\
\hline & Glucose & Hydrocortisone & Progesterone \\
\hline $\begin{array}{l}\text { Dilute solution } \\
\text { in normal saline }\end{array}$ & $14.0(4.0)$ & $5.4(2.1)$ & $6.3(1.2)$ \\
\hline $\begin{array}{l}\text { Externally added } \\
\text { solute to blank } \\
\text { DPPC liposomes }\end{array}$ & & $3.7(0.99)$ & \\
\hline $\begin{array}{l}\text { DPPC liposome- } \\
\text { entrapped solute }\end{array}$ & $0.006(0.001)$ & $3.0(2.8)$ & $4.6(0.34)$ \\
\hline
\end{tabular}

Standard deviation represents $3-4$ replicate experiments.

of glucose and hydrocortisone from the liposomes followed by an apparent zero-order release. The slope of the plot for glucose is $147 \mathrm{cpm} / \mathrm{h}(r=0.78)$ whereas the release rate for hydrocortisone was $42 \mathrm{cpm} / \mathrm{h}(r=0.78)$. The scatter in the data is primarily attributed to the experimental sensitivity in following the small changes of the radiolabeled solute in the bulk liquid with time; less than $5 \%$ of the drug was released in $60 \mathrm{~h}$. There was no measurable amount of progesterone outside the liposomes over the entire course of the experiment.

Fig. 3 shows the appearance of permeant with time for progesterone, hydrocortisone and glucose applied on the skin as a dilute solution in normal saline or as DPPC liposomally entrapped system. It is significant that, for blank DPPC liposomes containing radioactive lipid, no lipid could be detected in the receiver chamber up to $48 \mathrm{~h}$. As can be seen in Table 1, the effective permeability coefficients of the liposomally entrapped steroids are essentially identical to the permeability coefficient of the free steroid in aqueous solution. However, liposomal entrapment of glucose results in a permeability coefficient which is several orders in magnitude smaller than that for the free solute case.

\section{TABLE 2}

PERMEABILITY COEFFICIENTS OF LIPOSOME-ENTRAPPED PROGESTERONE IN VARYING LIPID COMPOSITION

\begin{tabular}{ll}
\hline Liposomal lipid & Permeability coefficients \\
composition & $\times 10^{4}(\mathrm{~cm} / \mathrm{h})($ S.D.) \\
\hline DMPC & $1.75(0.20)$ \\
DPPC & $4.60(0.34)$ \\
DSPC & 6.80 \\
DSPC : CHOL $(2: 1)^{*}$ & $6.70(0.60)$ \\
DPPC: $:$ DCP $(9: 1)^{*}$ & $9.00(0.37)$ \\
DPPC :SA $(9: 1)^{*}$ & $5.20(0.42)$ \\
\hline
\end{tabular}

\footnotetext{
* The ratio is on a molar basis.
} 
To explore the influence of liposomal charge and lipid composition on per zutaneous transport, progesterone was entrapped in DMPC, DSPC and DPPC lipos smes in combination with dicetylphosphate or stearylamine. The results of these ex periments are found in Table 2 . It is notable that the permeability coefficients are seen to be relatively indifferent to the composition of the liposomes.

\section{Discussion}

\section{Physicochemical properties of the liposomes}

Interaction of encapsulated drugs with the lipid component of the liposomes may alter the physicochemical properties of the liposomes which, in turn, would influence the drug transfer from the liposomes and the bioavailability of the drug. Differe atial scanning calorimetry is useful in characterizing physical properties of bilayers by following the lipid phase transitions (Ladbrooke and Chapman, 1969; Cater e al.. 1974). The transition temperature profile not only provides information as to possible 'fluidization' or 'solidification' of the bilayer, but also allows for the determination of critical thermodynamic properties (temperature range and enth alpy of transition). It also indicates whether the whole bilayer or part of it undergces a change. The very fact that the phase transition profile of a lipid bilayer is mod fied by an additive is direct proof of perturbation of the bilayer organization (Jain and Wu, 1977).

Glucose, which presumably concentrates exclusively in the aqueous compartments of the liposomes (Bangham et al., 1965; Demel et al., 1968; Papahadjopoulos e al., 1971), is without effect on the thermograms, indicating that it does not perturt the fundamental crystallinity of the bilayer. In contrast, progesterone and hydrc zortisone at a total concentration of $0.1 \%$ induce readily observable changes in the thermograms indicating that they are associated with the bilayer. The $37^{\circ} \mathrm{C}$ tem serature of the skin permeation experiments lies below the main transition temperature where the DPPC hydrocarbon chains 'melt' even in the presence of progesterone and

TABLE 3

SUMMARY OF PERMEABILITY COEFFICIENTS AND MECHANISMS

\begin{tabular}{llll}
\hline $\begin{array}{l}\text { Permeability } \\
\text { coefficient } \\
\text { (cm/hr) }\end{array}$ & Glucose & Hydrocortisone & Progesterone \\
\hline$P_{\mathrm{e}}$ & $6 \times 10^{-7}$ & $4 \times 10^{-4}$ & $4.6 \times 10^{-4}$ \\
$\mathrm{P}_{\mathrm{s}}$ & $14 \times 10^{-4}$ & $5.4 \times 10^{-4}$ & $6.3 \times 10^{-4}$ \\
$\mathrm{P}_{\mathrm{ht}}$ & $6.2 \times 10^{-4}$ & $3.7 \times 10^{-4}$ & 0 \\
$\mathrm{P}^{*}$ & $\sim 0$ & $3.95 \times 10^{-4}$ & $4.6 \times 10^{-4}$ \\
Mechinism & Free solute & $-99 \%$ liposome/skin & $100 \%$ liposome/skin \\
& (100\% liposome & solute transfer- & solute transfer- \\
& controlled; $\sim 1 \%$ free & controlled \\
& coleased- & solute-controlled & \\
\hline
\end{tabular}


hydrocortisone. Those liposomes containing cholesterol would also be expected to be of the solid type (Ladbrooke and Chapman, 1969). Thus, all transport experiments were carried out with 'gel phase' or 'solid' liposomes. It seems that liposome crystallinity per se may be of little consequence here as DMPC liposomes containing progesterone have transition temperatures well below $37^{\circ} \mathrm{C}$ and progesterone permeates skin from these liposomes at somewhat similar rates found with the solid liposomes (Table 3).

\section{Skin permeation of liposomal systems}

The failure of radiolabeled phospholipid in blank and hydrocortisone-entrapped liposomes to reach the receiver compartment shows unequivocally that these liposomes do not pass intact through the hairless mouse skin. It suggests that DPPC molecules are so locked into the liposomal structure that no appreciable free phospholipid exists to partition into the skin and permeate by this mechanism. The alternative explanation is that free DPPC is incapable of permeation which, although not tested, appears improbable because of its small molecular size and other physicochemical properties unfavorable for permeation.

Although liposomes clearly do not pass through the skin, they do induce remarkably different permeation behaviors of solutes entrapped within them. It appears that lipophilic drugs, like progesterone and hydrocortisone, pass through the skin with comparable facility to free drug (comparable mass transfer coefficients). Since the soluble payloads of lipophilic drugs can be increased through liposomal incorporation, more total drug may be delivered through skin via liposomes relative to simple aqueous solutions. Addition of 'empty' liposomes to free hydrocortisone in normal saline had no apparent influence on the permeation of the drug indicating that entrapment of the drug by liposomes is an important factor.

The preponderance of evidence does not support fusion of the liposomes to the skin surface as a mechanism of solute transport. Firstly, neither phospholipid molecules nor entrapped glucose molecules (other than the free glucose) appear on the receiver side. Secondly, the permeation of progesterone is not very dependent on liposome fluidity or charge as would be expected for fusion mechanism. If fusion was a dominant mechanism, the effective permeability of entrapped glucose should approach the values of the free solute, where in fact the permeability coefficient for entrapped glucose is about 3 orders of magnitude lower than that of free glucose. In our studies, the charge on the liposomes appears to have little influence on the overall permeation process with skin, although it has been reported to influence corneal penetration (Schaeffer and Krohn, 1982).

\section{Theoretical analysis}

The results of the foregoing permeation studies with DPPC liposomes containing model solutes (glucose, hydrocortisone and progesterone) suggests 3 probable mechanisms (see Appendix and Fig. 4): (1) release of liposome-entrapped solute and percutaneous absorption of the free solute: (2) release of liposome-entrapped solute 


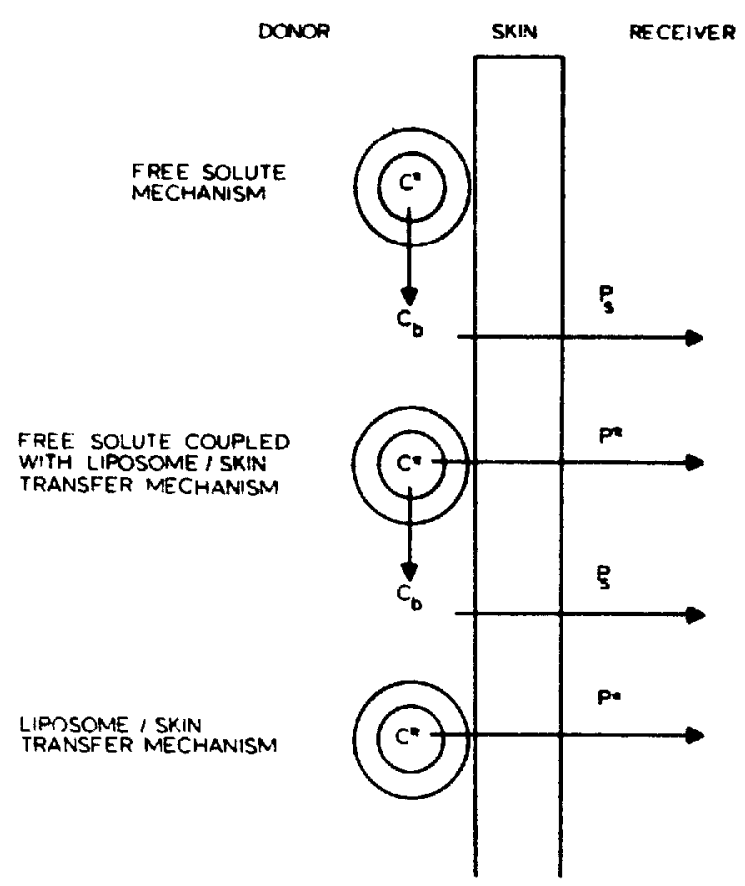

Fig. 4. Schematic description of various mechanisms in the skin permeation of liposome-entr pped solutes. Liposomes are not absorbed intact nor fused uith stratum corneum. See text for definitions if the permeability coefficients.

coupled with skin permeation of free solute and also direct liposome/skin silute transfer; and (3) skin permeation involving liposome/skin solute transfer. The first mechanism would be expected to apply to glucose, a hydrophilic solute entrappid in the aqueous phase of the liposomes: while the proposed third mechanism migl t be appropriate for progesterone, a hydrophobic solute located principally in the lipid bilayers.

\section{Glucose}

The skin permeability coefficient. $P_{s}$, for glucose in aqueous solution was $\mathrm{Frevi-}$ ously found to be $1.4 \times 10^{-3} \mathrm{~cm} / \mathrm{h}$, while the effective permeability coefficien . $P_{e}$. for the liposome-entrapped glucose and skin was $6 \times 10^{-7} \mathrm{~cm} / \mathrm{h}$. With the $u$ of Eqn. A-8 in the Appendix, it is seen that

$$
\frac{a A}{3 \varepsilon V_{D} P_{h 1}}=\frac{1}{P_{s}}-\frac{1}{P_{s}}=\frac{1}{6 \times 10^{-7}}
$$

In other words. release of glucose from the liposomes is the rate-determini..g st $p$ in the overall skin permeation kinetics of glucose. Subsequently, the mass tra isfer coefficient, $\mathbf{P}_{\mathrm{bl}}$, for glucose across the bilayers of the multilamellar liposomes $\mathrm{ci} \mathbf{n}$ be readily calculated. With $A=0.785 \mathrm{~cm}^{2}, \varepsilon=0.075, V_{D}=0.2 \mathrm{ml}$ and average $\mathrm{a}=0.4$ $\times 10^{-4} \mathrm{~cm}, P_{\mathrm{bl}}$ is $4.2 \times 10^{-10} \mathrm{~cm} / \mathrm{h}$. 
In vitro release studies indicate that the kinetics are very slow (Fig. 2). From the slope. $P_{b 1}$ is estimated by Eqn. A-22 to be $6.2 \times 10^{-9} \mathrm{~cm} / \mathrm{h}$ and, when compared to $4.2 \times 10^{-10} \mathrm{~cm} / \mathrm{h}$ obtained from the skin permeation studies, the in vitro $P_{\mathrm{hl}}$ is an order of magnitude larger. The disparity in $P_{h 1}$ by a factor of 15 takes on less significance in light of the 2000 -fold reduction in the effective skin permeability coefficient for the entrapped glucose as compared to free glucose. Thus, it is concluded that the experimental and theoretical studies are supportive of the free solute mechanism for the skin permeation of liposomally entrapped glucose wherein the release rate from the multilamellar liposome is the rate-determining step. It should be noted that even a 50 -fold smaller value for $P_{s}$ found in less hydrated skin in our laboratories has negligible effect on overall kinetics and mechanistic interpretation of skin transport of this polar substance in liposomes.

\section{Progesterone}

The skin permeability of progesterone from the liposome system $\left(P_{c}=4.6 \times 10^{-4}\right.$ $\mathrm{cm} / \mathrm{h})$ is nearly the same as that for the dilute aqueous solution $\left(P_{s}=6.3 \times 10^{-4}\right)$. In determining the mechanism the free solute absorption model used to analyze the glucose permeation studies is initially assumed. Upon employing Eqn. A-8, $P_{h 1}$ is estimated to be $1.2 \times 10^{-6} \mathrm{~cm} / \mathrm{h}$, which predicts that the permeability of the lipid bilayers should be larger than that of glucose. Contrary to this prediction, the in vitro release kinetics of progesterone intercalated in the bilayers was immeasurably slow (Fig. 2). Consequently, this suggests a direct liposome/skin transfer mechanism in the skin permeation of liposomally entrapped progesterone.

In performing the calculations for $P_{n\}}$. the average radius of $0.4 \mu \mathrm{m}$ used for the glucose-entrapped liposomes was also used here for progesterone. Insofar as the effect of additives on liposomal size is concerned, small amounts of uncharged substances $(<15 \%)$, which enter and accumulate within the bilayer structure, do not affect the morphological features of multilayer vesicles including particle size (Bangham et al., 1965; Sessa and Weissmann, 1968). Furthermore. polar additives which concentrate in the aqueous compartments also are without effect on liposomal morphology except when the liposomes are under the influence of very large osmotic stress (Weissmann et al., 1965). The $1 \%$ glucose (hypotonic) in the present studies falls well out of the osmotic differential necessary to alter liposomal size.

\section{Hydrocortisone}

The skin permeability of liposomally entrapped bydrocortisone is $4 \times 10^{-4} \mathrm{~cm} / \mathrm{h}$. which is essentially identical to the progesterone cise $\left(P_{c}=4.6 \times 10^{-4} \mathrm{~cm} / \mathrm{h}\right)$, and the permeability of hydrocortisone from aqueous: solution is $5.4 \times 10^{-4} \mathrm{~cm} / \mathrm{h}$. Furthermore, in vitro studies indicate slow leakage rates from the multilamellar liposomes. Here, $P_{h 1}$ is about $3.7 \times 10^{-4} \mathrm{~cm} / \mathrm{h}$. which is approximately three-fold slower than that found for glucose and. on the other extreme. more pronounced as compared to progesterone. Taken together, these findings suggest that both the free solute and liposome/skin transfer mechanisms are operating. With the aid of Eqn. A-13 and the aforementioned permeability coefficient values, $\mathrm{P}^{*}$ is found to be $3.95 \times 10^{4} \mathrm{~cm} / \mathrm{h}$. Thus, about $99 \%$ of the hydrocortisone permeates the skin by the 
liposome/skin solute transfer pathway and the small remainder by the free olute mechanism within which it is liposome release rate-controlled. The fact that an illary experiments utilizing a dilute volume fraction of liposomes containing hydrc cortisone $(\varepsilon=0.038)$ gave the same $P_{e}$ values as the higher liposome concentratior: case $(\varepsilon=0.075)$ provides additional support to the liposome/skin transfer mechani im as the principal transport pathway. If the free solute mechanism was highly inv lved. one would expect $P_{e}$ to be a function of the ratio of free solute to total olute concentration.

The overall process of liposome/skin solute transfer and permeation of both steroids are seen to occur with about equal facility. This mechanism is support ed by a study involving the permeation of liposomally entrapped, radiolabeled chole sterol and $\beta$-sitosterol across a silicone rubber membrane in a well-stirred, two-chi mber diffusion cell (Kreuter et al., 1981). Their results suggested a concerted twi-step process involving a 'collision complex' followed by a transfer of solute thro igh a thin hydrophilic layer into the membrane surface to explain the overall phenc mena and differences in permeability coefficients of cholesterol and the more hydrop nobic $\beta$-sitosterol. In our diffusion cell system the liposomes of low volume friction $(\varepsilon=0.075)$ are in a low volume, unstirred liquid and presumably, all have settl $x d$ on the skin membrane. The existence of a thin hydrophilic layer between the lipc some and skin cannot be entirely excluded.

\section{General remarks}

This theoretical analysis is summarized in Table 3. It sheds light on unders anding why liposomes are able to enhance the skin permeation rates of a steroid be yond that for the steroid in aqueous solution. In general, the permeation rate per unit area is equal to the product of the effective permeability coefficient and the applied concentration, i.e.

$J_{s}=P_{e} C$

While $P_{e}$ for the liposomally entrapped steroid may be somewhat smaller than $]_{e}$ for the free steroid in aqueous solution, one gains by the many-fold higher solubility in liposomes (Arrowsmith et al., 1983). The aqueous solubilities for the two steroids are only $12 \mu \mathrm{g} / \mathrm{ml}$ for progesterone and $285 \mu \mathrm{g} / \mathrm{ml}$ for hydrocortisone. The interf acial transfer occurs for liposomally entrapped steroids by 'collision complex trar sfer' between the steroid intercalated in the liposomal bilayer and the surface phas $s \mathrm{~s}$ of the stratum corneum.

In contrast to hydrophobic solutes entrapped in liposomes (aqueous and lipid regions), it is expected that hydrophilic solutes, which are entrapped principal y in the aqueous spaces of liposomes, would have significantly lower permeation ates than its aqueous solution counterpart. Here, the skin uptake rates will depend la gely upon the free solute concentration which is governed by the very slow leakage ates from liposomes. The interfacial transfer of the free solute involves stritum corneum/water partitioning. 
From a mechanistic and thermodynamic point of view, our theoretical analyses are consistent with the general observations of Mezei and Singh (1983) and Schaeffer and Krohn (1982) that corneal permeability of hydrophobic solutes is enhanced when they are liposomally entrapped and that the permeation of hydrophilic solutes is not enhanced by liposomal delivery.

\section{Appendix: physical models}

A variety of physical models is shown in Fig. 4 and will be developed. The models assume that the liposomes are not absorbed intact nor fused with the membrane. The liposomes are either unilamellar or multilamellar and monodispersed in size. Sink conditions are also assumed.

Free solute mechanism

This model involves the leakage of liposome-entrapped solute into the bulk aqueous medium and skin permeation of the free solute.

The release rate of solute from the liposomes is described by:

$\mathbf{J}_{1 \mathrm{ip}}=4 \pi \mathrm{a}^{2} \mathrm{nP}_{\mathrm{h} 1}\left(\mathrm{C}^{*}-\mathrm{C}_{\mathrm{h}}\right)$

where $J_{\text {lip }}$ is the release rate, mass $/ \mathrm{h} ; \mathrm{C}^{*}$ and $\mathrm{C}_{\mathrm{b}}$ are the solute concentrations in the liposomes and buik aqueous medium, respectively, mass $/ \mathrm{cm}^{3} ; \mathrm{P}_{\mathrm{bl}}$ is the permeability coefficient of the solute for the lipid bilayer(s), $\mathrm{cm}, / \mathrm{h}$; a is the radius of the liposome. $\mathrm{cm}$; and $\mathbf{n}$ is the total number of liposomes.

The flux of free solute across the skin into the sink of the receiver compartment is:

$\mathrm{J}_{\mathrm{AP}} \mathrm{AP}_{\mathrm{h}}$

Here, $J_{G}$ is the skin permeation rate, mass $/ h_{;} P_{s}$ is the permeability coefficient of the skin, $\mathrm{cm} / \mathrm{h}$; and $\mathrm{A}$ is the diffusional area of the skin, $\mathrm{cm}^{2}$.

Because of the steady-state continuity of mass flow, $J_{\text {lip }}=J_{s}$. Hence,

$C_{h}=\frac{4 \pi a^{2} n P_{h 1} C^{*}}{A P_{S}+4 \pi a^{2} n P_{h 1}}$

whereupon the substitution of $C_{b}$ into Eqn. A-2 yields:

$J_{4}=\frac{1}{\frac{1}{4 \pi a^{2} n P_{h i}}+\frac{1}{A P_{4}}} \cdot C^{*}$ 
The number of relatively monodispersed size liposomes is approximated by

$\mathrm{n}=\frac{\varepsilon \mathrm{V}_{\mathrm{D}}}{\mathrm{V}_{\text {lip }}}=\frac{3 \varepsilon \mathrm{V}_{\mathrm{D}}}{4 \pi \mathrm{a}^{3}}$

where $V_{D}$ is the volume of liposome suspension on the donor side of the skin: $\varepsilon$ is the volume fraction of liposomes; and $V_{\text {lip }}$ is the volume of a liposome of rad us a. Furthermore, the skin permeation rate is equal to the appearance rate:

$J_{s}=v \frac{d C}{d t}$

wherein $\mathrm{C}$ is the concentration of permeant on the receiver side, mass $/ \mathrm{cm}^{3}: \mathrm{V}$ is the volume of liquid, $\mathrm{cm}^{3}$; and $\mathrm{t}$ is the time.

Therefore, Eqn. A-4 becomes

$V \frac{d C}{d t}=\frac{1}{\frac{a}{3 \varepsilon V_{D} P_{h 1}}+\frac{1}{A P_{s}}} \cdot C^{*}$

Upon rearrangement into a computational form to determine the effective pe meability coefficient of the system from the linear plot of the amount of peri neant appearing with time, one gets

$\frac{V}{A C^{*}}\left(\frac{\Delta C}{\Delta t}\right)=P_{c}=\frac{1}{\frac{a A}{3 E V_{D} P_{h 1}}+\frac{1}{P_{s}}}$

It is assumed in Eqn. A-8 that $C^{*}$ remains fairly constant throughout the exper mental period. As can be seen, there are two limiting situations. When

$\frac{a A}{3 \varepsilon V_{D} P_{h 1}} \gg \frac{1}{P}$

by more than a factor of 20 , relcase of solute from the liposomes is the rate-lir viting step in skin permeation. The other extreme is the skin permeability-controllea case. The $P_{h 1}$ and $P_{s}$ are experimentally accessible from skin permeation studies.

Concurrem free solute and direct lipasome / skin mechanism

The rate of percutaneous absorption of drug is expressed by the sum $c f$ the permeation rates attributed to the drug solute in the bulk aqueous medium anc also the direct transfer of solute between the liposome and skin.

$J_{s}^{\prime}=A_{S} C_{b}+A P^{*} C^{*}$ 
where $P^{*}$ is the effective permeability coefficient for the liposome/skin transfer of solute and permeation processes, and the other terms have been previously described.

Since the permeation rate of the solute in the bulk fluid is dependent upon the release of solute from the liposomes, it follows that:

$\mathbf{J}_{\text {lip }}=\mathbf{J}_{\mathbf{s}}$

where $J_{\text {lip }}$ is expressed by Eqn. A-1 and $J_{s}$ by Eqn. A-2. Consequently, the substitution of Eqn. $\Lambda-3$ into $\Lambda-10$ yields:

$\mathbf{J}^{\prime}=\left[\mathrm{P}^{*}+\frac{1}{\frac{\mathrm{A}}{4 \pi \mathrm{a}^{2} n \mathrm{P}_{\mathrm{bl}}}+\frac{1}{\mathrm{P}_{\mathrm{s}}}}\right] \mathrm{AC}^{*}$

or

$\frac{V}{A C^{*}}\left(\frac{\Delta C}{\Delta t}\right)=P_{e}=P^{*}+\frac{1}{\frac{a A}{3 \varepsilon V_{D} P_{b 1}}+\frac{1}{P_{b}}}$

An analysis of Eqn. A-13 shows that, in the event $\mathrm{P}^{*}$ is zero, the expression reduces to Eqn. A-8 for the free solute mechanism.

\section{Direct liposome / skin mechanism}

When percutaneous absorption of a hydrophobic compound from a liposome system is appreciable and the release kinetics into the bulk aqueous medium is insignificant, i.e. $\mathbf{P}_{\mathrm{bl}}$ equals zero, then the direct liposome/skin transfer mechanism is a likely possibility. For this case, Eqn. A-13 becomes

$$
\frac{\mathrm{V}}{\Delta \mathrm{C}^{*}}\left(\frac{\Delta \mathrm{C}}{\Delta \mathrm{t}}\right)=\mathrm{P}^{*}
$$

\section{In vitro release kinetics}

The rate of appearance of solute in the bulk aqueous phase from all liposomes under quasi-sink conditions is:

$V_{h} \cdot \frac{d C_{b}}{d t}=4 \pi a^{2} n P_{h 1} C^{*}$

where the terms have been previously defined (Chowhan et al.. 1972, 1973).

The total volume of the liposome suspension, $V$, is:

$\mathbf{V}=\mathbf{V}_{\mathrm{b}}+\mathbf{n} \mathbf{V}_{\text {lip }}$ 
and, in terms of volume fraction, the total volume of liposomes is:

$\mathrm{nV}_{\text {lip }}=\mathrm{eV}$

and the volume of the bulk liquid, $V_{h}$, is

$V_{b}(1-\varepsilon) V$

where $\varepsilon$ is the volume fraction of liposomes. It follows from Eqn. A-17 that the total number of liposomes is:

$n=\frac{3 \varepsilon V}{4 \pi a^{3}}$

When the solute concentration of the liposomes. $C^{*}$, has not changed appre iably with time, it may be determined by:

$\mathrm{C}^{*}=\frac{\mathrm{T}}{\mathrm{nV}_{\mathrm{lip}}}$

where $\mathbf{T}$ is the initial amount of liposome-entrapped solute.

The substitution of Eqns. A-16-20 into 15 gives the following zero-order e: pressions:

$\frac{d C_{b}}{d t}=\frac{3 P_{b 1} T}{a(1-\varepsilon) V}$

and

$C_{b}=C_{b}(0)+\left(\frac{3 P_{b 1} T}{a(1-\varepsilon) V}\right) t$

from which the permeability coefficient of the bilayer(s), $P_{h 1}$, can be determir ed.

\section{Acknowledgement}

Supported in part by a gift from Hoffmann-La Roche, Inc.. Nutley, NJ.

\section{References}

Arrowsmith, M., Hadgraft, J. and Kellaway, I.W., The in vitro release of steroids from liposome s. Int. J. Pharm., 14 (1983) 191-208.

Bangham, A.D., Standish, M.M. and Watkins, J.C., Diffusion of univalent ions across the lar ellae of swollen phospholipids. J. Mol. Biol., 13 (1965) 238. 
Cater, B.R., Chapman, D., Hawes, S.M. and Saville, J., Lipid phase transitions and drug interactions. Biochim. Biophys. Acta, 363 (1974) 54-69.

Chowhan, Z.T., Yotsuyanagi, T. and Higuchi, W.I., Model transport studies utilizing lecithin spherules I. Critical evaluations of several physical models in the determination of the permeability coefficient for glucose. Biochim. Biophys. Acta, 266 (1972) 320-342.

Chowhan. Z.T.. Yotsuyanagi. T. and Higuchi. W.I.. Model transport studies utilizing lecithin spherules II. Transport of 3-O-methyl-D-glucose in D-glucose solution. J. Pharm. Sci., 62 (1973) 221-224.

Demel, R.A., Kinsky, S.C., Kinsky. C.B. and van Deenen, L.L.M., Effects of temperature and cholesterol on the glucose permeability of liposomes prepared with natural and synthetic lecithins. Biochim. Biophys. Acta, 150 (1968) 655-665.

Jain, M.K. and Wu, N.M., Effect of small molecules on the dipalmitoyl lecithin liposomal bilayer III. Phase transitions in lipid bilayer. J. Membr. Biol., 34 (1977) 157-201.

Kreuter, J., Higuchi. W.I., Ganesan, M.G. and Weiner. N.D., Delivery of liposome menibrane-associated sterols through silastic membranes. Biochim. Biophys. Acta, 676 (1981) 118-121.

Ladbrooke, B.D. and Chapman. D., Thermal analysis of lipids, proteins and biological membranes. A review and summary of some recent studies. Chem. Phys. Lipids, 3 (1969) 304-367.

Mezei, M. and Gulasekharam, V., Liposomes-a selective drug delivery system for the topical route of administration I. Lotion dosage form. Life Sci., 26 (1980) 1473-1477.

Mezei, M. and Gulasekharam, V.. Liposomes-a selective drug delivery system for the topical route of administration: gel dosage form. J. Pharm. Pharmacol., 34 (1982) 473-474.

Mezei, M. and Singh, K., Ocular distribution of liposome encapsulated drugs. Biol. Cell., 47 (1983) 180.

Papahadjopoulos, D., Nir, S. and Ohki, S., Permeability properties of phospholipid membranes: effect of cholesterol and temperature. Biochim. Biophys. Acta, 266 (1971) 561-583.

Patel, H.M. and Ryman, B.E., Systemic and oral administration of liposomes. In: Liposomes: from Physical Structure to Therapeutic Application, Elsevier/North-Holland Biomedical Press, Amsterdam, 1981, Ch. 15.

Poste. G., Liposomes targeting in vivo: problems and opportunities. Biol. Cell. 47 (1983) 19-38.

Sessa. G. and Weissmann, G., Phospholipid spherules as a model for biological membranes. J. Lipid Res.. 9 (1968) 310-318.

Schaeffer, H.E. and Krohn. D.L., Liposomes in topical drug delivery. Invest. Ophthalmol. Vis. Sci., 22 (1982) 220-227.

Weissmann, G., Sessa. G. and Weissmann, S.. Effect of steroids and Triton X-100 on glucose-filled phospholipid/cholesterol structures. Nature (Lond.), 208 (1965) 649-651. 